\title{
1 Reducing blood culture contamination using an initial specimen diversion
}

\section{device.}

Department of Intensive Care ${ }^{1}$, Laboratory of Clinical Microbiology and Immunology ${ }^{2}$, Department of Internal

Medicine $\mathrm{B}^{3}$, and Infectious Disease Unit ${ }^{4}$, Shaare Zedek Medical Center, affiliated with the Hebrew University-

* These authors contributed equally to the manuscript.

Keywords: blood culture, Contamination, diversion device

Running title: Diversion device reduces culture contamination

Clinical relevance: This study investigates the effect of an initial specimen diversion device on the rate of

20 culture contamination in hospitalized patients. It finds that the device is associated with a significant reduction in

21 contamination. This intervention may result in a reduction in costs, antibiotic use and duration of hospitalization.

Word count: Abstract - 236 words; Introduction through Discussion - 1724 words. 


\section{Abstract}

25 False positive blood cultures result from contamination, consuming microbiological laboratory resources and 26 causing unnecessary antibiotic treatment and lengthened hospitalizations. Skin sterilization has been shown to

27 reduce contamination; however, bacteria do not only colonize the surface of human skin, but are also found in 28 deeper tissues, requiring additional techniques to reduce contamination. An initial specimen diversion device

29 diverts the initial 1-2 $\mathrm{ml}$ of blood so as to remove any potentially contaminated skin plug, thus potentially further

30 reducing culture contamination. The device has been associated with a reduction in culture contamination over

31 short study periods in certain populations. However, more study is needed to understand whether the effect

32 continues over longer periods of time and in hospitalized patients. Thus, in this prospective, controlled pragmatic

33 study, cultures were obtained from hospitalized patients using the initial specimen diversion device, with cultures

34 taken using standard methods serving as control. In total, 671 blood cultures were obtained: 207 cultures were

35 taken using an initial specimen diversion device, with 2 (1.0\%) contaminated cultures and 464 cultures were taken

36 without the device, with $24(5.2 \%)$ contaminated cultures $(\mathrm{p}<0.008)$. No significant difference was shown in the

37 rate of true positive cultures. Thus, use of a diversion device was associated with reduced culture contamination in

38 hospitalized patients over a six month period without concomitant reduction in true positive cultures. This

39 intervention may result in a reduction in costs, antibiotic use and duration of hospitalization. 


\section{Introduction}

Bloodstream infections cause significant morbidity and mortality and their prompt identification is an essential part of modern medicine. Blood cultures, first described in the latter part of the nineteenth century ${ }^{1}$, are an essential element of the diagnosis and treatment of patients with such infections. However, as with all medical testing, false positive results occur and these result in delays in diagnosis, inappropriate treatment and significantly added expenses. ${ }^{2,3}$ False positive results in blood cultures have been described for as long as there have been such cultures and are primarily due to contaminants. ${ }^{1,2}$ It has been estimated that up to $50 \%$ of positive blood cultures represent contamination., ${ }^{2,3}$ These false positive cultures, at the microbiological laboratory level, require significant additional resources for workup. In addition, and perhaps more importantly, these false positive cultures may result in unnecessary antibiotic treatment as well as additional hospitalization days, causing needless harm to patients. All this together results an estimated additional cost of $\$ 4,385-\$ 8,720$ per false positive blood culture in the United States. ${ }^{3-6}$

Various methods have been implemented in order to reduce blood culture contaminants. Overall, combined interventions have proven effective in attenuating blood culture contamination and many institutions have successfully reducing blood culture contamination to the currently recommended rate of less than $2-3 \%$, with the highest achieving institutions having rates as little as $0.6 \% .{ }^{4,7}$ However, it has been shown that the bacteria which colonize the human skin are found not only on the surface but in fact in deeper tissues as well. It has further been shown that these bacteria cannot be completely removed even under ideal conditions. ${ }^{7,8}$ Thus zero or near zero blood culture contamination using current methods may be impossible, even with perfect technique. As such, it is reasonable to seek techniques beyond skin sterilization and improved adherence in order to further reduce the rate of blood culture contamination.

Recently, a new device was developed that diverts the initial $1-2 \mathrm{ml}$ blood so as to remove any potential skin plug with contaminants from entering the blood culture bottle. The SteriPath device (Magnolia Medical Technologies, Seattle, WA) is a closed-system, sterile blood collection system that diverts $1-2 \mathrm{~mL}$ of the initial venipuncture blood into an isolated diversion chamber and then allows venous blood to flow into culture bottles. 
culture contamination without reducing sensitivity to true blood stream infection. A recent study showed the utility of this device in an emergency room setting. ${ }^{9}$ However, this study was conducted over a short period of time, did not evaluate in-patients and used paired cultures, rather than a pragmatic design. Thus this study was designed as a pragmatic trial to evaluate the use of the diversion device in reducing blood culture contamination over the period of approximately 6 months in an in-patient population.

\section{Methods}

\section{Study design}

This prospective, controlled, pragmatic study was conducted in the Shaare Zedek Medical Center, a 1000-bed university-affiliated general hospital in Jerusalem, Israel. The hospital includes all major departments and services, including four medical and two geriatric wards, hematology and oncology, pediatrics, a surgical division including a vascular surgery unit, gynecology and obstetrics, cardiothoracic surgery, urology, orthopedics, plastic surgery, ophthalmology, otorhinolaryngology, neurosurgery and several intensive care units. The study was conducted in the 6-month period from March 2017 through August 2017 in one of the departments of medicine (Medicine B) in our institution.

During study period, as is standard practice in our institution, blood cultures were, in preference, taken by phlebotomy teams using venipuncture, two interventions that have been shown in multiple studies to reduce the rate of blood culture contamination. ${ }^{2,6}$ The remainder of cultures were taken mostly by resident physicians. Prior to study initiation a feedback mechanism was initiated in our institution in order to reduce contamination prior to intravenous line placement, by attaching the device to the newly placed intravenous catheter. The control group

91 consisted of blood cultures taken in the department using standard devices - Vacuette Holdex ${ }^{\circledR}$ (Grenier Bio-One,

92 Kremsmünster, Austria) attached to either a scalp vein set or to an intravenous catheter. In either method, blood 
94 Franklin Lakes, NJ). An automated blood culture system, BD BACTEC FX (BACTEC) was used to process the

95 blood cultures. Relevant characteristics of the blood culture, including date of culture and site of blood collection,

96 were recorded. Isolates were identified using MALDI-TOF (Vitek MS, bioMérieux) and/or standard biochemical

97 methods.

98 Blood culture contamination was identified by microbiological criteria - growth of coagulase-negative 99 staphylococci (CNS), corynebacteria, micrococci or alpha-hemolytic streptococci. Cultures could be reclassified 100 as true infections if such an organism was isolated from multiple blood cultures obtained by different 101 venipuncture. It should be noted that given the high sensitivity and short time to positivity of modern blood 102 culture systems ${ }^{10}$, time to positivity is not an effective method of identifying culture contaminants. If a single 103 blood culture showed growth of true infection and contaminant organisms, it was included in both categories.

104 During the first month of the study observers provided technical support to ensure proper use of the device by 105 the users - whether phlebotomists or physicians. The observers did not provide direct assistance in blood culture 106 collection. For the remaining five months of the study the device was used without observer presence. During the 107 entire period, blood cultures were collected according to the clinical discretion of the treating physician.

108 Ease of use of the device was evaluated using a standardized survey of phlebotomists and others involved in 109 sample collection.

110 Data was analyzed using Win-Pepi (version 11.65). Proportions were compared using the $\chi^{2}$-score or Fisher's 111 exact test, where appropriate. Continuous variables were compared by Student's t-test or Mann-Whitney112 Wilcoxon test where appropriate. Two-tailed $\mathrm{p}$ values were taken and a $\mathrm{p}$ value $<0.05$ was defined as significant. 113 The study was approved by the Institutional Review Board of Shaare Zedek Medical Center. 


\section{Results}

117 In the 6 months of the study period, 671 blood cultures were obtained in the study department. Of these, 207

118 cultures were recorded to have been taken using the initial specimen diversion device; this population served as

119 the study group. In 464 cultures, use of the device was not recorded; this population served as the control group. A

120 significant difference in age was found between the control group and the study group (74 \pm 1.4 versus $77 \pm 2.1$,

121 respectively, $\mathrm{p}<0.004)$. Otherwise no differences between the groups were noted (Table 1).

Of the 464 cultures in the control group, 68 (14.7\%) were positive for bacterial growth. Of those positive cultures, 24 were defined as contaminants, thus resulting in a contamination rate of $5.2 \%$. In the study group, 207 blood cultures were obtained. Of these, $18(8.7 \%)$ were positive for bacterial growth. Of these positive cultures, 2 were defined as contaminants, thus resulting in a contamination rate of $1.0 \%$ in the study group ( $\mathrm{p}<0.008$ for the difference between the groups). With the exception of a single culture in the control group which grew Streptococcus parasanguinis, all other contaminated cultures grew coagulase-negative Staphylococci.

Of the control group cultures, 44 were defined as true positives resulting in a true positive rate of $9.5 \%$. Of the study group cultures, 16 were defined as true positives resulting in a true positive rate of $7.7 \%$. Enterobacteriaceae and staphylococci were the most common causes of true bacteremia in both groups. No significant difference in the true positive rate or in the microbiological characteristics of true positive cultures were noted between the groups (Table 2 and Figure 1).

In a survey of ease of device use, all respondents who had used the device more than once considered it 134 slightly or moderately more difficult to use than standard practice (grade 2 or 3 out of 5).

\section{Discussion}

A substantial fraction of positive blood cultures represent contamination, rather than true bloodstream

137 infection ${ }^{2,3}$, including in the control group of our study (Figure 1), where 24 out of $464(5.2 \%)$ cultures 138 represented contamination and $44(9.5 \%)$ represented true infection. These false positive cultures, at the 139 microbiological laboratory level, require significant futile resources for workup. In addition, and perhaps more 
importantly, these false positive cultures result in unnecessary antibiotic treatment as well as excessive

141 hospitalization days, causing needless harm to patients. All this together results in substantial additional costs per

142 false positive blood culture..$^{3-6}$

143 In this study, we investigated the efficacy of an initial specimen diversion device in reducing the rate of blood

144 culture contamination. We found that the device was associated with a reduction in blood culture contamination 145 with a $5.2 \%$ (24 out of 464 cultures) contamination rate in the control group and a $1.0 \%$ (2 out of 207 cultures) 146 contamination in the study group $(\mathrm{p}<0.008)$. These results are consistent with those obtained in a recent study 147 performed in an emergency room population. ${ }^{9}$ In our study, the control group was noted to be younger than the 148 study group.

This study suggests that use of an initial specimen diversion device can effectively reduce culture contamination among hospitalized patients without impacting the rate of true positive cultures. Furthermore, this reduction can be maintained over a relatively extended period of time.

The study has several limitations. First, though this study was controlled, a pragmatic design using a convenience sample, rather than a randomized control, was employed. Such a study is limited in controlling for 154 confounders related to selection of sample. Indeed, the study population was older than that of the control, though no other significant differences were noted (Table 1). Additionally, this study was a single center study with a relatively small study population. As such, results should be interpreted cautiously.

In conclusion, this study found that use of an initial specimen diversion device was associated with a reduction 158 in blood culture contamination in hospitalized patients over a six month period - from 5.2\% (24/464) in the 159 control group to $1.0 \%(2 / 207-\mathrm{p}<0.008)$ in the study group - without a concomitant reduction in true positive 160 cultures. This is an intervention that can result in a reduction in costs, unnecessary antibiotic use and duration of 161 hospitalization. 


\section{Acknowledgments}

164 The authors would like to thank Dr. David Raveh for assistance in statistical analysis.

165 The funding source, Magnolia Medical Technologies, provided the SteriPath device as well as initial technical

166 support in their use. The funding source did not provide any other financial support nor were they involved in

167 study design; the collection, analysis and interpretation of data; in the writing of the report; or in the decision to 168 submit the article for publication. 


\section{References}

1. White FW. Cultures from the blood in septicemia, pneumonia, meningitis and chronic diseases. J Exp Med $1899 ; 4: 425-450$.

2. Snyder SR, Favoretto AM, Baetz RA, Derzon JH, Madison BM, Mass D, et al. Effectiveness of practices to reduce blood culture contamination: A Laboratory Medicine Best Practices systematic review and metaanalysis. Clin Biochem 2012;45:999-1011.

3. Bates DW, Goldman L \& Lee TH. Contaminant blood cultures and resource utilization. The true consequences of false-positive results. JAMA 1991;265:365-369.

4. Bekeris LG, Tworek JA, Walsh MK \& Valenstein PN. Trends in blood culture contamination: a College of American Pathologists Q-Tracks study of 356 institutions. Arch Pathol Lab Med 2005;129:1222-1225.

5. Ramirez P, Gordón M, Cortes C, Villarreal E, Perez-Belles C, Robles C, et al. Blood culture contamination rate in an intensive care setting: Effectiveness of an education-based intervention. Am J Infect Control 2015;43:844-847.

6. Gander RM, Byrd L, DeCrescenzo M, Hirany S, Bowen M \& Baughman J. Impact of blood cultures drawn by phlebotomy on contamination rates and health care costs in a hospital emergency department. J Clin Microbiol 2009;47:1021-1024.

7. Hall KK \& Lyman JA. Updated review of blood culture contamination. Clin Microbiol Rev 2006;19:788802.

8. Brown E, Wenzel RP \& Hendley JO. Exploration of the microbial anatomy of normal human skin by using plasmid profiles of coagulase-negative staphylococci: search for the reservoir of resident skin flora. J Infect Dis 1989;160:644-650.

9. Rupp ME, Cavalieri RJ, Marolf C \& Lyden E. Reduction in blood culture contamination through use of initial specimen diversion device. Clin Infect Dis 2017;65:201-205. 

aCC-BY-ND 4.0 International license.

193 10. Zadroga R, Williams DN, Gottschall R, Hanson K, Nordberg V, Deike M, et al. Comparison of 2 Blood 194 Culture Media Shows Significant Differences in Bacterial Recovery for Patients on Antimicrobial Therapy. 195 Clin Infect Dis 2013;56:790-797.

196 


\section{Tables}

199

Table 1. Characteristics of study population. CI: confidence interval

\begin{tabular}{lccc}
\hline Variable & $\begin{array}{c}\text { Diversion device } \\
\mathbf{n = 2 0 7}(\mathbf{\%})\end{array}$ & $\begin{array}{c}\text { Standard practice } \\
\mathbf{n = 5 0 6}(\boldsymbol{\%})\end{array}$ & $\begin{array}{c}\text { p- } \\
\text { value }\end{array}$ \\
\hline Male & $118(57.0)$ & $311(61.5)$ & 0.446 \\
Age - years $\pm 95 \%$ CI & $77 \pm 2.1$ & $74 \pm 1.4$ & 0.004 \\
\hline $\begin{array}{l}\text { Place of residence } \\
\quad \text { home }\end{array}$ & $148(71.5)$ & $385(76.1)$ & 0.292 \\
$\quad$ assisted living & $11(5.3)$ & $23(4.5)$ & 0.847 \\
$\quad$ chronic care facility & $48(23.2)$ & $98(19.4)$ & 0.303 \\
Hospitalized in last year & $128(61.8)$ & $304(60.1)$ & 0.669 \\
Seven day mortality & $36(17.4)$ & $77(15.2)$ & 0.498 \\
\hline
\end{tabular}


Table 2. Microbiological and clinical characteristics of positive cultures. (CoNS: coagulase negative staphylococci)

\begin{tabular}{lccc}
\hline Variable & $\begin{array}{c}\text { Diversion device } \\
\mathbf{n = 2 0 7}(\boldsymbol{\%})\end{array}$ & $\begin{array}{c}\text { Standard practice } \\
\mathbf{n = 4 6 4}(\boldsymbol{\%})\end{array}$ & $\begin{array}{c}\mathbf{p}- \\
\text { value }\end{array}$ \\
\hline All positive cultures & $18(8.7)$ & $68(13.4)$ & 0.034 \\
\hline Culture contamination & $2(1.0)$ & $24(4.7)$ & 0.008 \\
$\quad$ CoNS & $2(1.0)$ & $23(4.5)$ & 0.013 \\
\hline Other & $0(0.0)$ & $1(0.2)$ & 1.000 \\
Contamination by age group & & & \\
\hline $18-64$ & $0 / 28(0.0)$ & $2 / 96(2.1)$ & 1.000 \\
\hline $65-80$ & $1 / 70(1.4)$ & $16 / 226(7.1)$ & 0.081 \\
\hline $81+$ & $1 / 109(0.9)$ & $6 / 184(3.3)$ & 0.253 \\
\hline True positive & $16(7.7)$ & $44(8.7)$ & 0.558 \\
\hline Enterobacteriaceae & $3(1.4)$ & $20(4.0)$ & 0.067 \\
\hline Staphylococci & $12(5.8)$ & $18(3.6)$ & 0.312 \\
\hline Other & $1(0.5)$ & $6(1.2)$ & 0.447 \\
\hline
\end{tabular}




\section{Figures}

205

Figure 1. Positive culture rate for both contaminated and true positive cultures.

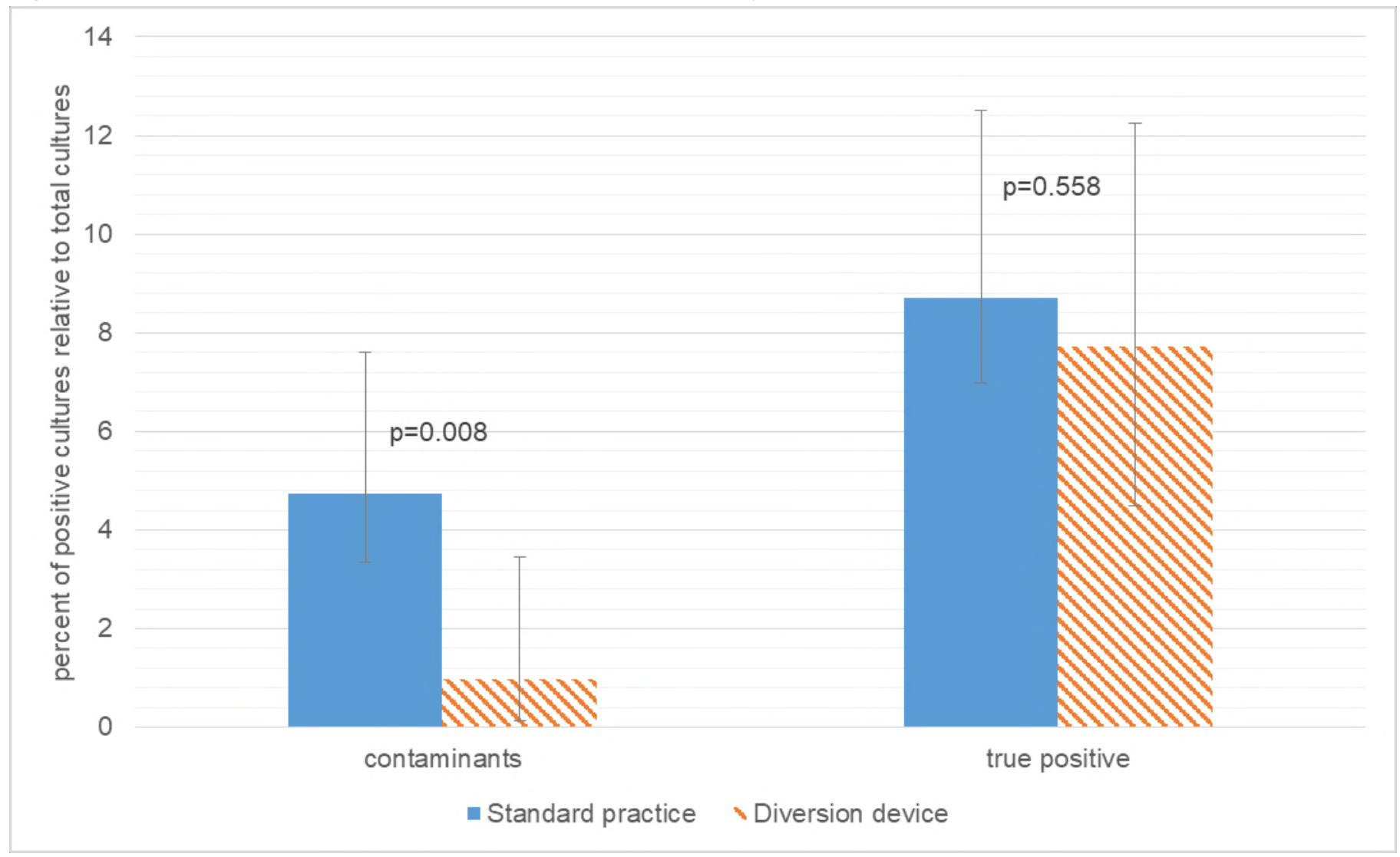




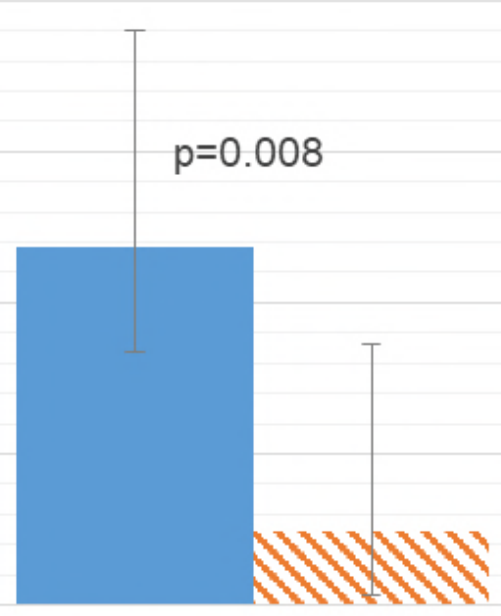

contaminants

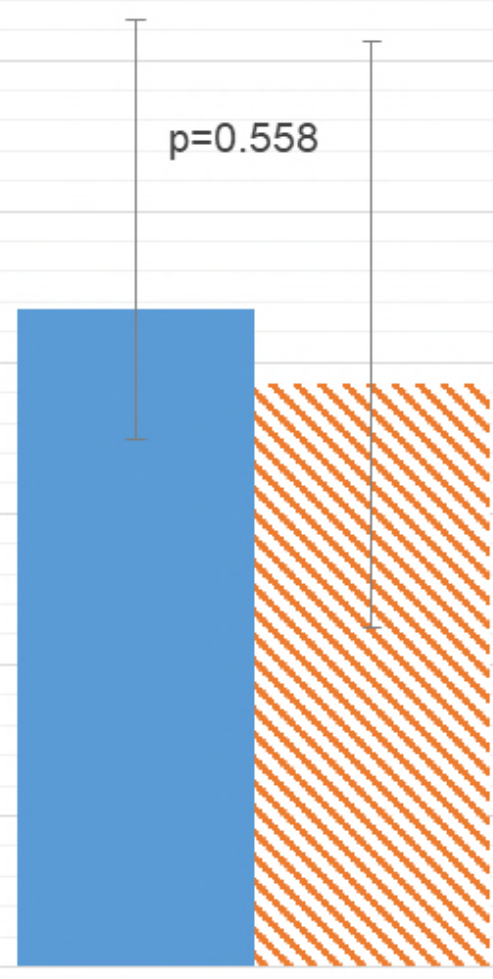

true positive

- Standard practice Diversion device 